\title{
Eficacia de los ejercicios de estabilización lumbopélvica en pacientes con lumbalgia
}

\section{Efficacy of lumbopelvic stabilization exercises in patients with lumbalgia}

\author{
Varela-Esquivias A, * Díaz-Martínez L, ${ }^{\ddagger}$ Avendaño-Badillo D ${ }^{\S}$
}

Centro de Rehabilitación del Instituto Guanajuatense para Personas con Discapacidad.

RESUMEN. Introducción: El dolor lumbar es una de las principales causas de incapacidad laboral en México; dentro del tratamiento conservador, se incluye la realización de ejercicios para la disminución del dolor. Objetivo: Valorar los efectos en la disminución del dolor lumbar con un programa controlado de ejercicios diseñados para mejorar la flexibilidad y fuerza en el núcleo o centro del cuerpo (core). Material y métodos: Se incluyeron a 18 personas: 12 mujeres y seis hombres de entre 24 y 70 años con dolor lumbar, el cual estaba referido con una puntuación de 2 a 10 utilizando la escala visual análoga (EVA), con o sin dolor en la extremidad inferior. Se aconsejó a los participantes abstenerse de tomar medicamentos, alcohol y de realizar actividades físicas intensas durante el período de tratamiento. Los participantes realizaron 12 sesiones de 10 ejercicios en tres series de 10 repeticiones, tres veces por semana, evaluándose durante estas sesiones: peso, distancia dedos piso, fuerza de recto anterior, diámetro abdominal e índice de Oswestry inicial y final. Resultados: Los datos se analizaron con la prueba de Wilcoxon. Se evidenció disminución no sólo en el dolor ( $p<0.01)$, sino una mejoría en el peso, la flexibilidad y la percepción de incapacidad por la escala de Oswestry $(\mathrm{p}<0.01)$, mostrando que la serie de ejercicios propuestos es una alternativa para al manejo del dolor lumbar. Discusión: Se recomienda la presencia de un terapeuta que acompañe y corrija las posturas para que los ejercicios sean bien realizados, así como darle valor al grado de incapacidad que produce el dolor en el desempeño cotidiano de las personas que lo presentan.

Palabras clave: Dolor lumbar, discapacidad, Oswestry, ejercicios de estabilización lumbar.
ABSTRACT. Introduction: Low back pain is one of the main causes of work disability in Mexico, conservative treatment includes performing exercises. Objective: To assess the effects of lower back pain with a controlled exercise program to improve flexibility and strength in the core or center of the body. Material and methods: We included 18 people, 12 women and six men between 24 and 70 years of age, with low back pain, with a score of 2 to 10 using the visual analogue scale (VAS), with or without referred pain in the lower limb. Participants were advised to refrain from taking medications, alcohol and intense physical activities during the treatment period. The participants carried out 12 sessions of 10 exercises in 3 sets of 10 repetitions, 3 times per week, evaluating: weight, distance toes, anterior rectum strength, abdominal diameter, initial and final Oswestry index. Results: The results were analyzed with the Wilcoxon test, showing a decrease not only in pain $(\mathrm{p}<0.01)$, but an improvement in weight, flexibility and perception of disability due to the Oswestry scale $(p<0.01)$ showing that the series of exercises proposed is an alternative to the management of low back pain. Discussion: It is recommended the presence of a therapist who accompanies and corrects the postures so that the exercises are performed well, as well as giving value to the degree of disability caused by pain in the daily performance of the people who present it.

Keywords: Low back pain, disability, Oswestry, lumbar stabilization exercises.

\section{Nivel de evidencia: IV}

* Licenciada en Terapia Física. Universidad de Guanajuato. Guanajuato, México.

‡ Medicina de Rehabilitación. Centro de Rehabilitación. Instituto Guanajuatense para Personas con Discapacidad. Guanajuato, México.

$\S$ Medicina de Rehabilitación. Servicio de Rehabilitación Ortopédica, Instituto Nacional de Rehabilitación «Luis Guillermo Ibarra Ibarra». Ciudad de México, México.

Dirección para correspondencia:

LTFR Aranza Esquivias-Varela

Parque Guanajuato Bicentenario, puerta 2, Km 3.5, Carretera de cuota Silao-Guanajuato.

El Capulín, Silao de la Victoria, Guanajuato, México. Teléfono: 47211791 30, ext. 102.

E-mail: a.esquiviasvarela@ugto.mx. 


\section{Introducción}

El dolor lumbar es una de las principales causas de incapacidad laboral en México. ${ }^{1}$ Para tratar de disminuir el dolor, el tratamiento conservador incluye la realización de ejercicios. Para aquellos pacientes con dolor lumbar e hiperlordosis, se usan los tradicionalmente conocidos ejercicios de Williams y, para aquellos pacientes con rectificación de la lordosis lumbar, los ejercicios de McKenzie.

No obstante, debido a la confusión que esto crea, tanto en su prescripción como en la enseñanza de estos ejercicios, actualmente se prescriben ejercicios que fortalecen y mejoran la flexibilidad del centro o centro del cuerpo (core) y la musculatura antigravitatoria; éstos son conocidos como ejercicios de estabilización lumbopélvica (EELP), ${ }^{2,3,4}$ los cuales son muy variados y deben ajustarse de acuerdo con la edad y las condiciones de enfermedades asociadas de las personas, ya que, por ejemplo, las personas con gonartrosis no pueden realizar algunas posiciones, por lo que éstas deben ajustarse o modificarse para poderse ejecutar. ${ }^{5}$

La relación entre la musculatura del tronco y la musculatura lumbopélvica sirve para establecer un nexo para la función, pero su alteración biomecánica es la principal contribuyente al dolor. ${ }^{5,6}$ Los EELP se basan en el entrenamiento de fuerza y flexibilidad del centro del cuerpo (core), el cual está formado por cuatro grupos musculares: al frente, el recto abdominal; a los lados, los oblicuos; en la parte posterior, los paraespinales, el dorsal ancho, el cuadrado lumbar, la psoas mayor, la psoas menor y los glúteos; finalmente, en la parte superior el diafragma y en la parte inferior la musculatura del suelo pélvico, isquiotibiales y una porción de refuerzo del recto anterior.

El centro del cuerpo (core) participa en el control de la posición y el movimiento del tronco sobre la pelvis lo que transfiere la fuerza y el movimiento a las extremidades superiores e inferiores. ${ }^{7,89}$ Éste tiene tres subsistemas: pasivo, activo y neuromuscular. El subsistema pasivo se refiera a aquellas estructuras que carecen de capacidad contráctil, pero ayudan a la estabilidad articular del cinturón lumbopélvico. Éste se compone de huesos, de articulaciones, de cápsula articular, ligamentos y de cartílagos y discos. El subsistema activo es propiamente la musculatura del centro del cuerpo (core), los tendones y las fascias, es decir, los elementos contráctiles. Finalmente, el subsistema neuromuscular es aquél formado por la inervación local, baro y mecanorreceptores que se encargan de responder a las variaciones captadas y realizar las correcciones necesarias ante situaciones repentinas, organizando patrones de movimiento compensatorios. Asimismo, éste es el encargado de ajustar todo el conjunto, recibiendo la información del sistema pasivo y activo.

Si la persona es capaz de estabilizar, reeducar y fortalecer el centro del cuerpo (core), será capaz de prevenir lesiones o mejorar los tiempos de rehabilitación y recuperación, tanto a nivel funcional como deportivo. ${ }^{10}$

Por ello, el ejercicio físico es una terapia usada para reducir el dolor y la limitación funcional en las personas con dolor lumbar. ${ }^{2,3,8,10}$ Sin embargo, existen pocos estudios que determinen el número de repeticiones y la frecuencia necesaria para producir un efecto adecuado y duradero para el control del dolor. ${ }^{11,12,13,14}$ Por esta razón, este estudio pretende valorar un programa específico de ejercicios de estabilización lumbopélvica que mejoren la flexibilidad y fuerza del centro del cuerpo (core) para que se reduzca la sintomatología dolorosa a nivel lumbar. Así, se evaluaron los efectos en la fuerza abdominal, el peso, el perímetro abdominal, la distancia dedos-piso y la escala de incapacidad por dolor lumbar de Oswestry - un cuestionario específico para dolor lumbar que mide las limitaciones que el dolor produce en las actividades cotidianas-.

\section{Material y métodos}

El comité de ética institucional aprobó el estudio, el cual se adhirió a las pautas establecidas por la Declaración de Helsinki. De esta manera, se realizó un estudio prospectivo, longitudinal, experimental y de medidas repetidas para comparar los efectos después del programa de ejercicios.

En este estudio participaron 18 personas: 12 mujeres y seis hombres de entre 24 y 70 años de edad con dolor lumbar, quienes fueron reclutados en el Departamento de Fisioterapia. Todos presentaban dolor lumbar leve a intenso con una puntuación evaluado de dolor promedio de 2 a 10 a través de la escala visual análoga (EVA). Se incluyeron a los pacientes incluso si habían tenido dolor referido o compromiso neurológico en extremidades inferiores. Se aconsejó a los participantes que se abstuvieran de tomar medicamentos, alcohol y actividades físicas intensas durante el período de tratamiento. Se excluyeron del estudio a mujeres embarazadas, personas con hipertensión descontrolada, con trastornos cognitivos y que faltaran tres veces consecutivas al programa.

A cada participante se le dio una plática inicial en donde se les explicó en qué consistía el estudio; se les mostraron imágenes de los ejercicios, esquemas anatómicos de cómo está formado el centro del cuerpo (core), se les entregó impreso el programa de ejercicios y se obtuvo su firma de consentimiento informado para su inclusión.

El cálculo de la muestra se realizó con el programa EPI INFO $^{\circledast}$ versión 7.2.2.6, donde se consideró: nivel de confianza de $95 \%$, el valor de la significación de $5 \%$ y la potencia de la prueba de $80 \%$, obteniéndose una muestra mínima de 14 participantes.

Todos los participantes fueron evaluados al inicio y final del programa por un médico especialista en medicina de rehabilitación, de donde se registró: el peso, el perímetro abdominal, la EVA, la fuerza abdominal y el puntaje del cuestionario de Oswestry, ${ }^{15}$ el cual mide cómo el dolor limita o repercute en las actividades diarias de la persona por medio de 48 preguntas divididas en nueve secciones: 1) intensidad del dolor; 2) cuidado personal; 3) levantar peso; 4) caminar; 5) sentarse; 6) estar de pie; 7) sueño; 8) vida social y 9) vida sexual. 
Los participantes realizaron, de manera presencial, 12 sesiones de 10 ejercicios en series de 10 repeticiones de cada uno, tres veces por semana siempre con acompañamiento del investigador principal para corregir posturas y vigilar la correcta realización de los ejercicios. El programa consistió en: calentamiento, enseñanza de respiración profunda, enseñanza para la identificación del centro del cuerpo (core) y enseñanza propia de cada uno de los ejercicios con medidas de corrección postural para hacerlos adecuadamente. A continuación, se describe en qué consistió cada uno de los apartados y cada uno de los ejercicios:

Calentamiento: Se estableció como necesario para preparar la musculatura y, con ello, evitar lesiones. Se realizó durante 2 o 3 minutos, en el que se incluyeron:

1. Movilizaciones de cuello (flexión, extensión y rotaciones).

2. Movilizaciones en todos los rangos de movimiento de miembros superiores e inferiores.

3. Ejercicios de flexión de ambas caderas y rodillas con la espalda bien recta.

4. Saltar o trotar suavemente sobre el mismo sitio durante 15 segundos.

Respiración: mediante una inhalación profunda y lenta por la nariz, se desciende el diafragma sin compensar con el tórax; a continuación se sigue de una exhalación lenta por la nariz, mientras se contrae abdomen para elevar diafragma.

Identificación del centro del cuerpo (core): para esto, se dio una sesión de enseñanza, mostrándoles los esquemas anatómicos. A todos los pacientes se les pidió tocar o identificar visualmente y en ellos mismos las zonas que se explicaban.

Medidas de corrección postural: se indicó que debían evitar encorvarse; en caso de que por otro problema corporal, por ejemplo, artrosis de rodillas, se les dificultara colo-
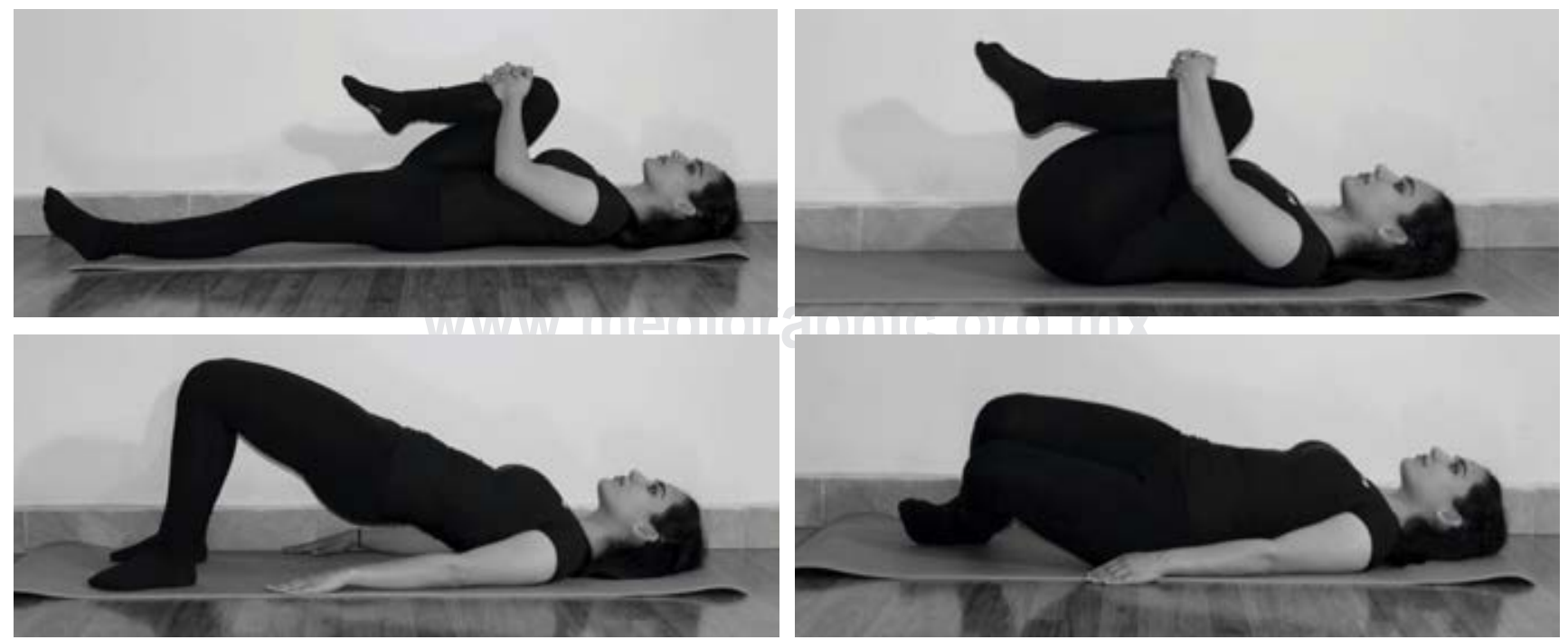

carse en las posiciones adecuadas para los ejercicios, se les indicó sujetarse de una silla o solicitar asistencia para pararse y sentarse o utilizar una almohadilla lumbar para sostener la curvatura de la espalda baja.

\section{Programa de ejercicios}

Se les indicó hacer los ejercicios en series de 10 repeticiones de tres series de cada uno. Entre serie y serie, se le pidió a cada paciente tomar un tiempo de reposo contando despacio hasta 5. Cada ejercicio consistió en:

Ejercicio 1. Sobre el piso boca arriba, con piernas estiradas, se flexiona alternadamente una rodilla hacia el pecho sosteniéndola por cinco segundos de cada lado.

Ejercicio 2. Sobre el piso boca arriba, con piernas estiradas se flexionan ambas rodillas llevándolas al pecho ayudándose con las manos. Sostener durante cinco segundos.

Ejercicio 3. Sobre el piso boca arriba, con los brazos sobre el piso, hay que levantar la pelvis durante tres segundos y volver a la posición inicial.

Ejercicio 4. Sobre el piso boca arriba, se hace una inhalación por la nariz, seguida de una exhalación mientras se intenta pegar la zona lumbar al suelo al tiempo que se contraen los glúteos. Después, con las piernas flexionadas y juntas, se balancean de un lado a otro (Figura 1).

Ejercicio 5. En cuatro puntos, hay que alinear el ancho de las palmas de las manos con el ancho de rodillas. Posteriormente, hay que inhalar viendo hacia arriba y marcar la curvatura de la lordosis lumbar; exhalar mirando hacia abajo, invirtiendo la curvatura.

Ejercicio 6. En cuatro puntos, con las manos apoyadas en el piso y con los codos en extensión, con la cabeza en posición neutra, se extienden alternando pierna y brazo contrario al mismo tiempo. En caso de no poderse reali-

Figura 1: Ejercicios del 1-4. 

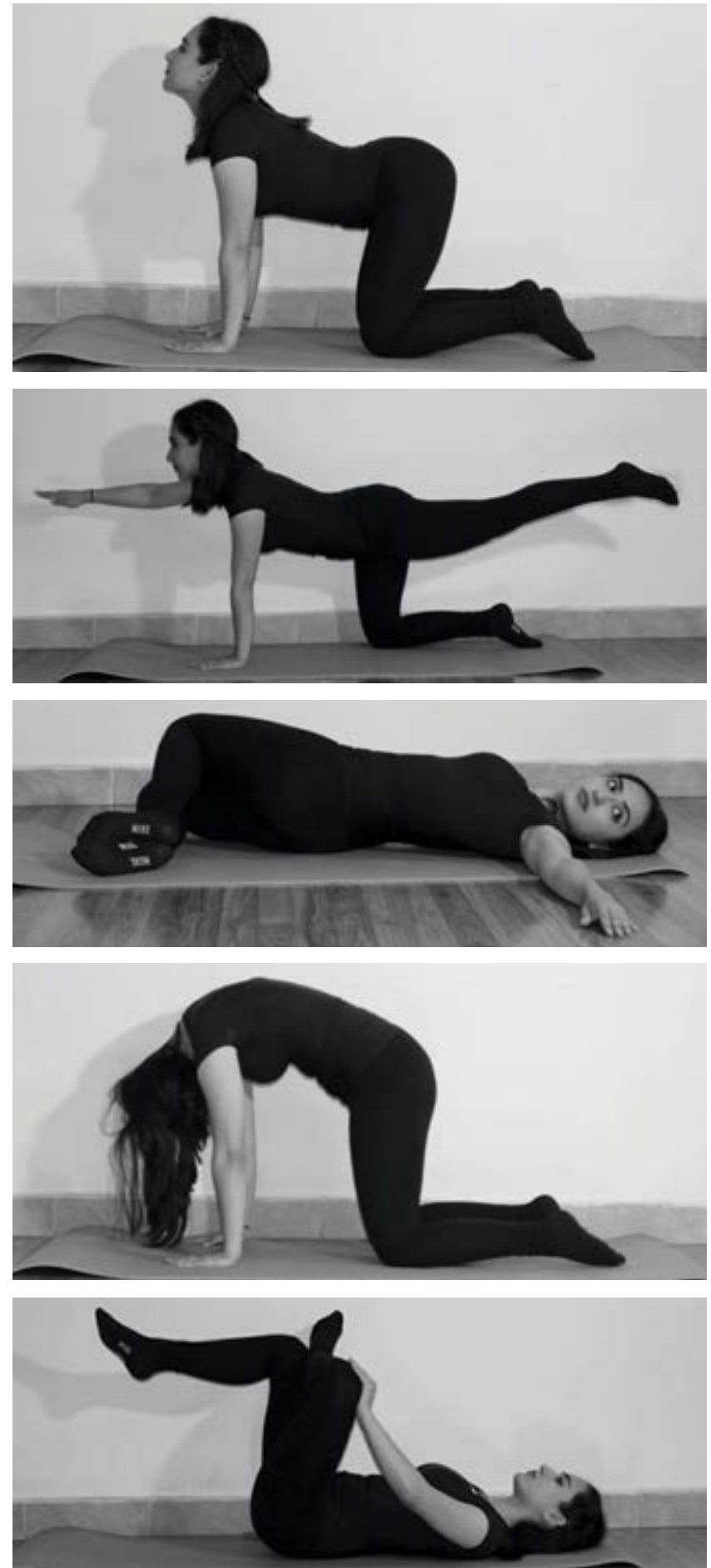

Figura 2: Ejercicios del 5-8.

zar en el piso apoyado en cuatro puntos, se puede hacer en bipedestación apoyado de una silla y seguir el mismo patrón.

Ejercicio 7. En el piso en posición decúbito supino, con una pierna flexionada, se coloca la pierna contraria sobre la otra, mientras que la pierna flexionada se levanta del piso para que forme un ángulo de $90^{\circ}$. Con el brazo del lado de la pierna sobrepuesta, se empuja la pierna hacia adelante lo más posible. Mantener la posición durante 15 segundos y cambiar de lado.

Ejercicio 8. En el piso boca arriba, con los brazos extendidos a los lados, flexionar ambas piernas y balancearlas hacia a un lado, en tanto que la cabeza y cuello se balancean hacia el lado contrario. Mantener la posición 15 segundos de cada lado (Figura 2).

Ejercicio 9. De rodillas, sentado sobre los talones y sin despegar glúteos de los talones, se estiran los brazos al frente con los codos en máxima extensión, intentando tocar con la frente el piso. Exhalar lentamente y mantener el máximo estiramiento durante 15 segundos.

Ejercicio 10. En el piso, en decúbito ventral, se colocan los glúteos lo más cerca a la pared y extender las extremidades inferiores hacia arriba sin flexionar las rodillas, haciendo dorsiflexión máxima de tobillos para mantener los músculos isquiotibiales en máxima extensión. Mantener la posición 15 segundos y descansar otros cinco segundos (Figura 3).

\section{Resultados}

Los datos de la muestra estudiada se muestran en la Tabla 1. Todos los pacientes presentaron mejoría en la percepción del dolor de acuerdo con la EVA $(\mathrm{p}<0.01)$
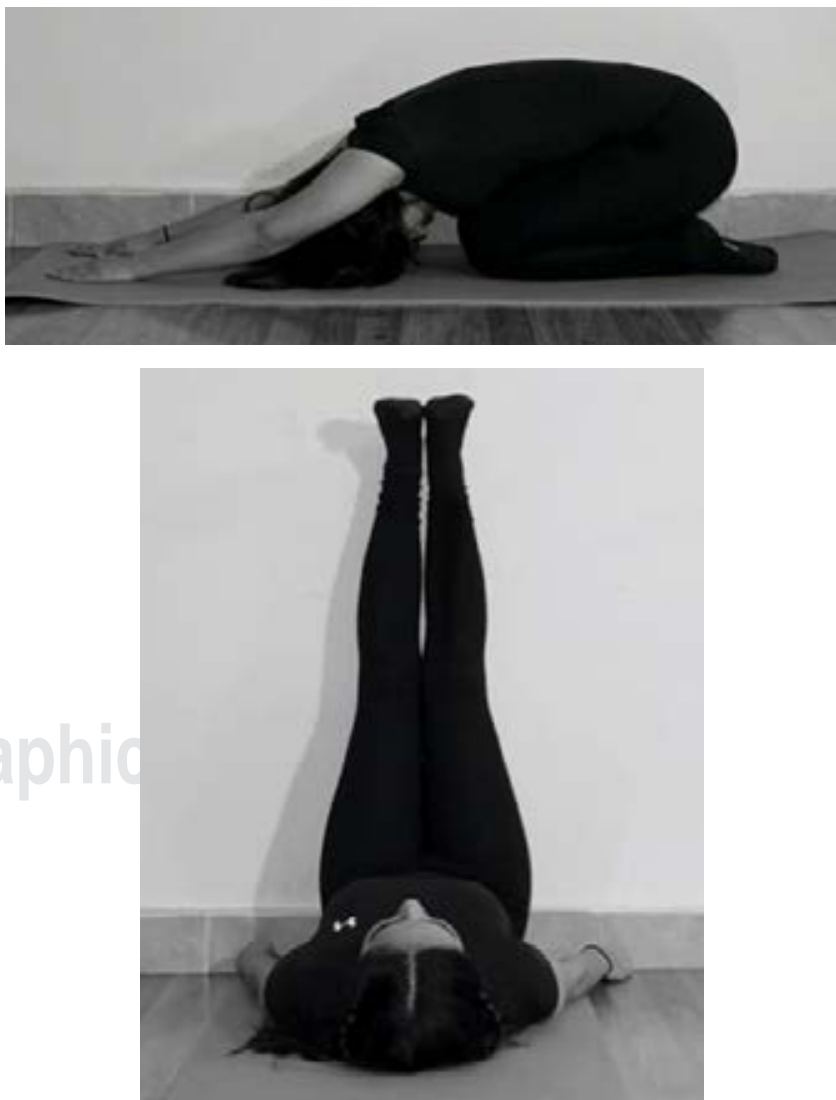

Figura 3: Ejercicios 9 y 10. 


\begin{tabular}{|c|c|c|c|c|c|c|c|c|c|c|c|c|c|c|}
\hline Género & $\begin{array}{c}\text { Edad } \\
\text { (años) }\end{array}$ & EVA 1 & EVA 2 & $\begin{array}{l}\text { Ddp1 } \\
(\mathrm{cm})\end{array}$ & $\begin{array}{l}\text { Ddp2 } \\
(\mathrm{cm})\end{array}$ & F1 & F2 & $\begin{array}{c}\text { Peso } \\
\text { inicial } \\
(\mathrm{kg})\end{array}$ & $\begin{array}{c}\text { Peso final } \\
(\mathrm{kg})\end{array}$ & $\begin{array}{c}\text { Talla } \\
(\mathrm{m})\end{array}$ & $\begin{array}{l}\text { Dal } \\
(\mathrm{cm})\end{array}$ & $\begin{array}{l}\mathrm{Da} 2 \\
(\mathrm{~cm})\end{array}$ & $\begin{array}{l}\mathrm{O} 1 \\
(\%)\end{array}$ & $\begin{array}{l}\mathrm{O} 2 \\
(\%)\end{array}$ \\
\hline $\mathrm{F}$ & 67 & 2 & 1 & 24 & 22 & 1 & 1 & 83.5 & 83 & 1.51 & 127 & 127 & 38 & 20 \\
\hline $\mathrm{F}$ & 41 & 6 & 4 & 31 & 26 & 1 & $1+$ & 73 & 72 & 1.61 & 92 & 91 & 54 & 38 \\
\hline $\mathrm{F}$ & 42 & 6 & 3 & 28 & 24 & 1 & $1+$ & 98 & 98 & 1.59 & 125 & 125 & 56 & 36 \\
\hline $\mathrm{F}$ & 39 & 8 & 5 & 9 & 3 & 1 & $1+$ & 71.5 & 69 & 1.48 & 96 & 93 & 60 & 20 \\
\hline $\mathrm{F}$ & 66 & 3 & 0 & 21 & 19 & 2 & $2+$ & 88.5 & 88 & 1.49 & 114 & 113 & 36 & 20 \\
\hline $\mathrm{F}$ & 58 & 6 & 2 & 20 & 17 & 3 & $3+$ & 61 & 58 & 1.48 & 92 & 89 & 54 & 20 \\
\hline $\mathrm{F}$ & 43 & 2 & 0 & 7 & 5 & $2+$ & 3 & 55 & 55 & 1.53 & 82 & 82 & 32 & 18 \\
\hline $\mathrm{F}$ & 70 & 5 & 2 & 30 & 28 & $1+$ & $1+$ & 66 & 65 & 1.52 & 101 & 99 & 52 & 22 \\
\hline $\mathrm{F}$ & 54 & 5 & 1 & 9 & 7 & $2+$ & 3 & 63 & 62 & 1.56 & 85 & 83 & 54 & 20 \\
\hline $\mathrm{F}$ & 59 & 6 & 4 & 20 & 18 & $1+$ & 2 & 60 & 60 & 1.5 & 88 & 88 & 56 & 22 \\
\hline $\mathrm{F}$ & 60 & 6 & 4 & 18 & 16 & 1 & $1+$ & 43.5 & 43.5 & 1.46 & 75 & 75 & 50 & 40 \\
\hline $\mathrm{F}$ & 42 & 5 & 2 & 8 & 6 & $2+$ & 3 & 60 & 58 & 1.6 & 85 & 83 & 38 & 18 \\
\hline M & 24 & 4 & 1 & 33 & 9 & 4 & $4+$ & 72 & 71 & 1.71 & 87 & 87 & 50 & 18 \\
\hline M & 65 & 2 & 1 & 32 & 15 & $1+$ & 2 & 115 & 113 & 1.8 & 125 & 124 & 30 & 28 \\
\hline $\mathrm{M}$ & 49 & 6 & 4 & 12 & 9 & 2 & 3 & 80 & 80 & 1.77 & 92 & 92 & 32 & 22 \\
\hline M & 51 & 7 & 4 & 30 & 12 & 1 & $1+$ & 79 & 77 & 1.75 & 105 & 104 & 50 & 38 \\
\hline $\mathrm{M}$ & 48 & 5 & 2 & 15 & 8 & $2+$ & $3+$ & 80 & 80 & 1.82 & 87 & 87 & 35 & 20 \\
\hline M & 63 & 5 & 3 & 28 & 17 & $1+$ & $2+$ & 85 & 84 & 1.56 & 101 & 101 & 48 & 37 \\
\hline
\end{tabular}

y en la flexibilidad de la zona lumbar de acuerdo con la distancia dedos-piso $(\mathrm{p}<0.01)$. Asimismo, hubo mejoría en el descenso del peso $(\mathrm{p}<0.01)$ y en las medidas del diámetro abdominal final $(\mathrm{p}<0.01)$ y en la percepción de incapacidad de Oswestry ( $<<0.01)$, aunque no en el promedio del índice de masa corporal inicial y final (Tabla 2). La fuerza no fue valorable, ya que, aunque existe mejoría, no se analizó porque se utilizó una medida subjetiva para graduar la fuerza.

En cuanto al grado de incapacidad para las actividades diarias de acuerdo con la percepción registrada por las personas a las que se aplicó la prueba, al inicio del tratamiento, tres pacientes mostraron un índice de incapacidad por dolor lumbar de acuerdo con la valoración de Oswestry moderado (21-40\% de limitación para realizar sus actividades diarias) y 11 pacientes evidenciaron un grado severo (41-60\% de limitación para realizar sus actividades diarias); pero, al final del estudio, nueve pacientes tenían un índice mínimo $(0-20 \%)$ y nueve moderado $(21-40 \%)$, siendo significativa

\begin{tabular}{|l}
\hline \multicolumn{3}{c}{ Tabla 2: Principales características de medición } \\
antes y después del programa.
\end{tabular}

la mejoría en la percepción general de independencia en sus actividades diarias $(\mathrm{p}<0.01)$ (Tabla 3$)$. Todos los pacientes que iniciaron el estudio lo terminaron adecuadamente.

\section{Discusión}

Este programa de ejercicios demostró ser efectivo para el manejo del dolor lumbar. En él, se evidencia la importancia de explicar detalladamente la realización de los ejercicios y el impacto que tiene sobre la mejoría del dolor y el movimiento en la disminución de la limitación en las actividades diarias.

Es útil enseñar al paciente sobre las estructuras involucradas en el mecanismo del dolor, ya que esto parece impactar positivamente en el apego al programa, ya que ningún paciente abandonó el tratamiento y todos siguieron las instrucciones dadas. Es recomendable que un terapeuta físico esté acompañando al paciente y corrigiendo las posturas durante el tratamiento, ya que esto impacta en los buenos resultados, aunque para confirmarlo se requiere un grupo en el que no se contara con el acompañamiento de un terapeuta y se analizara que pasa si los pacientes hacen el ejercicio por sí solos.

A pesar del conocimiento general del ejercicio como una alternativa no invasiva y efectiva para el tratamiento del dolor lumbar, aún no existe un consenso general sobre cuál es la evaluación óptima y cuál es la estrategia de intervención. ${ }^{15}$ Este estudio apoya la necesidad de que, para la realización de los ejercicios, hay que tomar en cuenta la condición física, las alteraciones agregadas (como la artropatía de rodillas o incluso la edad) y la motivación a los pacientes, pone énfasis en la necesidad de guiarlos 


\begin{tabular}{|c|c|c|c|c|c|}
\hline & \multicolumn{2}{|c|}{ Inicial } & & \multicolumn{2}{|c|}{ Final } \\
\hline & Personas & Promedio & & Personas & Promedio \\
\hline Moderada & 7 & 38.89 & Mínima & 9 & 50 \\
\hline Severa & 11 & 61.11 & Moderada & 9 & 50 \\
\hline Total & 18 & & Total & 18 & \\
\hline
\end{tabular}

y supervisarlos, para que, de esta manera, se asegure el rendimiento y la calidad de la ejecución de los ejercicios; además, señala la necesidad de que hay que guiar a los pacientes al utilizar pruebas adicionales en la valoración médica, como la escala de incapacidad para dolor lumbar de Oswestry, para medir el impacto del dolor en la vida diaria y no sólo para modificar el dolor, sino para impactar positivamente en la independencia y desempeño diario, ya que su utilización permite obtener información desde la perspectiva del paciente, conocer el grado de eficacia de las distintas técnicas de tratamiento empleadas y comparar los resultados con otros estudios. ${ }^{15,16}$

\section{Agradecimiento}

Agradecemos a todos los participantes del estudio. Un particular agradecimiento a la Dra. Diana Avendaño Badillo por su valiosa orientación para la realización de este estudio.

Bibliografía

1. Soto-Padilla M, Espinosa-Mendoza RL, Sandoval-García JP, GómezGarcía F. Frecuencia de lumbalgia y su tratamiento en un hospital privado de la Ciudad de México. Acta Ortop Mex. 2015; 29(1): 40-5.

2. Paungmali A, Joseph LH, Sitilertpisan P, Pirunsan U, Uthaikhup S. Lumbopelvic core stabilization exercise and pain modulation among individuals with chronic nonspecific low back pain. Pain Pract. 2017; 17(8): 1008-14.

3. Coulombe BJ, Games KE, Neil ER, Eberman LE. Core stability exercise versus general exercise for chronic low back pain. $J$ Athl Train. 2017; 52(1): 71-2.

4. Gomes-Neto M, Lopes JM, Conceição CS, Brasileiro A, Sousa C, Oliveira C, et al. Stabilization exercise compared to general exercises or manual therapy for the management of low back pain: A systematic review and meta-analysis. Phys Ther Sport. 2017; 23: 136-42.

5. Paulsen RT, Rasmussen J, Carreon LY, Andersen MØ. Return to work after surgery for lumbar disc herniation, secondary analyses from a randomized controlled trial comparing supervised rehabilitation versus home exercises. Spine J. 2020; 20(1): 41-7.

6. Miura T, Sakuraba K. Properties of force output and spectral EMG in young patients with nonspecific low back pain during isometric trunk extension. J Phys Ther Sci. 2014; 26(3): 323-9.

7. Karayannis NV, Jull GA, Hodges PW. Movement-based subgrouping in low back pain: synergy and divergence in approaches. Physiotherapy. 2016; 102(2): 159-69.
8. Sousa Filho LF, Barbosa Santos MM, de Farias Neto JP, da Silva Junior WM. The addition of neurodynamic exercises to extensionoriented exercises among patients with chronic back-related leg pain: a study protocol. J Bodyw Mov Ther. 2019; 23(3): 473-8.

9. Calatayud J, Escriche-Escuder A, Cruz-Montecinos C, Andersen LL, Pérez-Alenda S, Aiguadé R, et al. Tolerability and muscle activity of core muscle exercises in chronic low-back pain. Int J Environ Res Public Health. 2019; 16(19): 3509.

10. Stuge B. Evidence of stabilizing exercises for low back- and pelvic girdle pain- a critical review. Braz J Phys Ther. 2019; 23(2): 181-6.

11. Shahvarpour A, Gagnon D, Preuss R, Henry SM, Larivière C. Trunk postural balance and low back pain: Reliability and relationship with clinical changes following a lumbar stabilization exercise program. Gait Posture. 2018; 61: 375-81.

12. Almousa S, Lamprianidou E, Kitsoulis G. The effectiveness of stabilising exercises in pelvic girdle pain during pregnancy and after delivery: A systematic review. Physiother Res Int. 2018; 23(1): 10.1002/pri.1699.

13. Standaert CJ, Weinstein SM, Rumpeltes J. Evidence informed management of chronic low back pain with lumbar stabilization exercises. Spine J. 2008; 8: 114-20.

14. Brumitt J, Matheson JW, Meira EP. Core stabilization exercise prescription, part I: current concepts in assessment and intervention. Sports Health. 2013; 5(6): 504-9.

15. Alcántara-Bumbiedro S, Flórez-García MT, Echávarri-Pérez C, García-Pérez F. Escala de incapacidad por dolor lumbar de Oswestry. Rehabilitación (Madr). 2006; 40(3): 150-8.

16. Cortell-Tormo JM, Sánchez PT, Chulvi-Medrano I, et al. Effects of functional resistance training on fitness and quality of life in females with chronic nonspecific low-back pain. J Back Musculoskelet Rehabil. 2018; 31(1): 95-105.

Conflicto de intereses: los autores no tienen conflicto de intereses que declarar ni denunciar.

Consideraciones ético-legales: este protocolo de investigación está bajo los cuatro principios internacionales de la declaración de Helsinki, la confiabilidad, confidencialidad y la Ley General de Salud (en materia de investigación clínica). Para lo cual se consideró:

- Beneficencia. Modular el tono postural, mejorar la flexibilidad y postura, reeducar la función respiratoria y disminuir el dolor local e irradiado.

- No maleficencia. En todo momento, se cuidará la integridad de la salud del participante de la investigación, reduciendo los riesgos a los que puede ser susceptible.

- Autonomía. La participación del involucrado en este estudio es voluntaria y puede decidir no participar o cambiar su decisión y retirar el consentimiento en cualquier momento, sin dar ningún tipo de explicación, así como solicitar la destrucción de los datos obtenidos.

- Justicia. No existió discriminación o selección irregular de los participantes. 